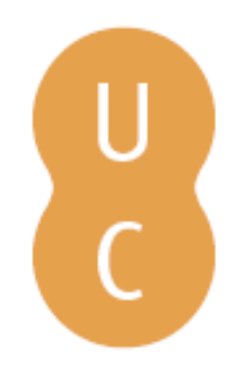

\title{
pommalina
}

\section{Die Selbstbezüglichkeit des Denkens bei Aristoteles}

Autor(es): Jung, Christian

Publicado por: Imprensa da Universidade de Coimbra

URL

persistente:

URI:http://hdl.handle.net/10316.2/31629

DOI:

DOI:http://dx.doi.org/10.14195/978-989-26-0205-9_9

Accessed : $\quad$ 26-Apr-2023 11:55:36

A navegação consulta e descarregamento dos títulos inseridos nas Bibliotecas Digitais UC Digitalis, UC Pombalina e UC Impactum, pressupõem a aceitação plena e sem reservas dos Termos e Condições de Uso destas Bibliotecas Digitais, disponíveis em https://digitalis.uc.pt/pt-pt/termos.

Conforme exposto nos referidos Termos e Condições de Uso, o descarregamento de títulos de acesso restrito requer uma licença válida de autorização devendo o utilizador aceder ao(s) documento(s) a partir de um endereço de IP da instituição detentora da supramencionada licença.

Ao utilizador é apenas permitido o descarregamento para uso pessoal, pelo que o emprego do(s) título(s) descarregado(s) para outro fim, designadamente comercial, carece de autorização do respetivo autor ou editor da obra.

Na medida em que todas as obras da UC Digitalis se encontram protegidas pelo Código do Direito de Autor e Direitos Conexos e demais legislação aplicável, toda a cópia, parcial ou total, deste documento, nos casos em que é legalmente admitida, deverá conter ou fazer-se acompanhar por este aviso.

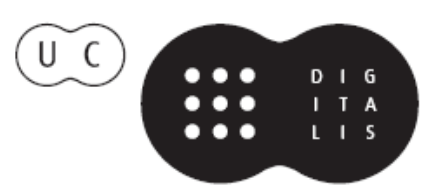


Edmundo Balsemão Pires

Burkhard Nonnenmacher

Stefan Büttner-von Stülpnagel

Editors

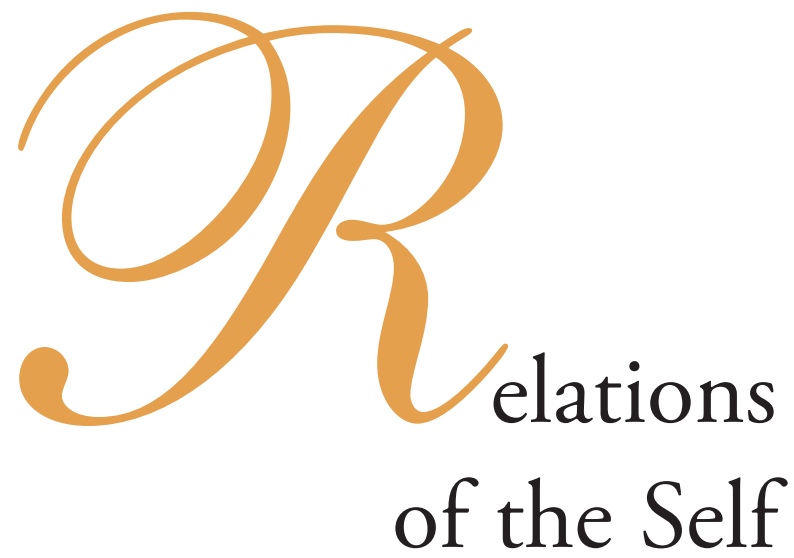




\section{Christian Jung \\ Wien}

\section{DIE SELBSTBEZÜGLICHKEIT DES DENKENS BEI ARISTOTELES}

\section{Einleitung}

Unter den Selbstbezügen, die für die philosophische Theoriebildung von Interesse sind, dürfte das Selbstbewusstsein eine unangefochtene Spitzenstellung einnehmen. Nichts hat die Philosophen spätestens seit dem Ende des Mittelalters mehr beschäftigt als das Phänomen der Subjektivität und ihr Verhältnis zu der Welt der Objekte. Die Beziehung des Menschen zu seiner Welt wurde dabei primär epistemisch gedeutet. Die im Vordergrund stehenden Fragen waren also: Was kann ich wissen? Und wer bin ich, der ich dieses Wissen habe? Wie kann ich mich selbst wissen? Als Ahnvater dieser subjektzentrierten Philosophie wird gemeinhin Descartes genannt. Die Legitimität dieses historiographischen Allgemeinplatzes wurde zu Recht bestritten, zuerst von Walter Schulz in seinem Buch Der Gott der neuzeitlichen Metaphysik (1957). Inzwischen liegen Untersuchungen zur Theorie der Subjektivität bei Nikolaus von Kues, Meister Eckhart und Dietrich von Freiberg vor, die deutlich machen, dass es auch vor Descartes einen philosophischen Begriff vom Subjekt gab. Der entscheidende Terminus ist im Rahmen der scholastischen Philosophie intellectus, den wir gewöhnlich entweder mit Intellekt oder Geist übersetzen. Da der Begriff des Geistes jedoch zu den missverständlichsten und missbrauchtesten der Philosophiegeschichte gehört, möchte ich lieber vom Intellekt sprechen. In jüngerer Zeit hat insbesondere die sog. „Philosophie des Geistes“ für eine allzu weite Dehnung des Begriffes gesorgt. In der mittelalterlichen Philosophie ist der Begriff intellectus die Übersetzung des griechischen voũc. Das ist natürlich in erster Linie durch die Aristoteles-Rezeption begründet, die seit den Kommentaren Alberts des Großen in Blüte stand. Zu den wirkmächtigsten Schriften des Aristoteles zählt der Traktat De anima, in dem er seine Naturphilosophie der Seele entfaltet. Dieser Traktat enthält auch seine wichtigsten Äußerungen zum Intellekt. Die mittelalterlichen Theorien der Subjektivität gehen teils unmittelbar, teils durch neuplatonische Schriften vermittelt auf die aristotelische Intellekttheorie zurück. Man kann sich mithin fragen, ob es bei Aristoteles selbst auch schon eine Theorie der Subjektivität gibt.

Dass die antike Philosophie eine Theorie des Selbstbewusstseins kennt, wird von den meisten Fachgelehrten eher bezweifelt. Und gewiss darf man nicht erwarten, dass die in der Neuzeit auf den Begriff gebrachten Fragestellungen im Wortlaut etwa bei Aristoteles zu 
finden sind. Die Frage muss also vielmehr lauten, ob die Sache des Selbstbewusstseins ihren Niederschlag in der Philosophie gefunden hat. Um - wenigstens im Ansatz - ein Antwort hierauf zu finden, wird man den Intellektbegriff bei Aristoteles untersuchen müssen. Auch gibt es Stellen in De anima, die auf eine Reflexivität des Sinnesvermögens hinweisen. Schließlich spricht Aristoteles dem Ersten Beweger des Kosmos eine geistige Natur zu, die bekanntermaßen

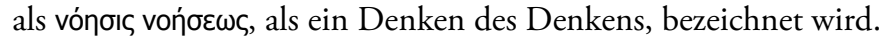

Um bei Aristoteles eine Theorie der Subjektivität rekonstruieren zu können, müssen wir in zwei Schritten vorgehen. Der erste Schritt muss erklären, in welchem Sinne Aristoteles sowohl beim Menschen als auch bei Gott von voũ sprechen kann. Denn wenn die

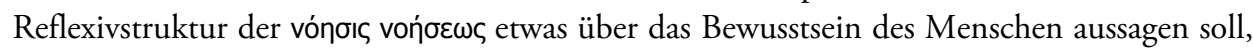
obwohl sie den göttlichen Intellekt charakterisiert, muss zunächst plausibel gemacht werden, dass, aristotelisch gedacht, der Intellekt als Intellekt bei Gott und Mensch derselbe ist - auch wenn Aristoteles selbst diese Identifikation noch nicht explizit vollzieht. In einem zweiten und für unsere Frage wichtigeren Schritt gilt es, die Bedeutung des Ausdrucks vónбıৎ

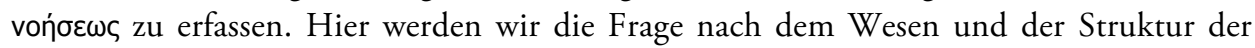
Subjektivität stellen müssen. Dabei werden wir sehen, dass Aristoteles eine Alternative zu dem neuzeitlichen Reflexionsmodell bieten kann.

\section{Die Göttlichkeit des menschlichen Intellekts}

Aristoteles entwickelt seine Psychologie als eine naturphilosophische Teildisziplin. Dabei geht er systematisch vor, indem er die Stufen organischen Lebens hinaufsteigt bis zum Menschen, genauer gesagt: indem er das für die jeweilige Stufe der scala naturae spezifische Seelenvermögen untersucht. An der Spitze dieser scala steht der Intellekt, durch den der Mensch artspezifisch ausgezeichnet ist. Die anderen Seelenvermögen sind entweder allen Lebewesen gemein (so das Nährvermögen) oder allen Tieren (die Sinnesvermögen). Die seelischen Leistungen eines Organismus bauen ebenso aufeinander auf wie die Teile des Organismus selbst. Jeder Lebensvollzug eines Körpers ist eine Aktivität der Seele, die ihrerseits zu diesem Vollzug in der Lage sein muss. Das ist der Sinn der Rede von Seelenvermögen oder - wie Aristoteles meistens sagt - Seelenteilen. Dabei achtet Aristoteles peinlich darauf, dass die Anzahl der Vermögen nicht unnötig vermehrt wird. So weist er beispielsweise dem Nährvermögen ebenfalls die Fähigkeit zur geschlechtlichen Zeugung zu, weil er einen engen physiologischen Zusammenhang zwischen Verdauung und Samenbildung sieht. Die wechselseitige Bezogenheit von Körper und Seele bedeutet nun, dass ein Organ nur dank eines es beseelenden und zu seiner eigentümlichen Leistung befähigenden Seelenvermögens arbeitet und dass umgekehrt jedes Seelenvermögen eine organische Grundlage haben muss. Die einzige Ausnahme hiervon ist der menschliche Intellekt. Er alleine von allen Seelenvermögen kommt Aristoteles zufolge ohne Organ aus. An diesem Punkt möchte ich in den Text von De anima einsteigen.

In Kapitel $\Gamma 4$ von De anima sagt Aristoteles, dass der Intellekt mit dem Körper nicht vermischt sein kann, weil er sonst gewisse aktuale Eigenschaften annehmen müsste. Das ist aber unmöglich, weil er dem Wesen nach reine Potentialität ist. Außerdem müsste er ein 
Organ haben wie das Sinnesvermögen. Aristoteles formuliert dies folgendermaßen: „Daher ist es auch nicht konsequent zu denken, dass sie [sc. die Vernunft] mit dem Körper vermischt sei; denn <dann> nähme sie wohl eine bestimmte Eigenschaft an, entweder kalt oder warm, oder sie hätte wohl auch ein [sc. körperliches] Organ, wie das Sinnesvermögen “. Aristoteles betrachtet den Intellekt als von den Sinnesvermögen dadurch verschieden, dass er ohne ummittelbare Daten aus der Welt der sinnlichen Objekte auskommt.

Doch freilich gibt Aristoteles auch zu, dass er ohne die Einbildungskraft, die pavtacía, keine Objekte hätte. Die pavtaría aber hängt aufs engste mit den von den Sinnesvermögen empfangenen Daten zusammen. Insofern braucht das Denken eine sinnliche Quelle. Aber

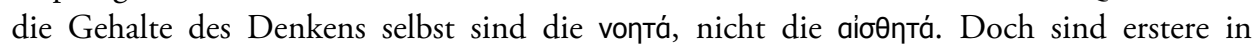
letzteren enthalten. D.h. dass die intelligiblen Formen in den sinnlichen Formen verborgen sind und dass der Intellekt so beschaffen ist, dass er diese intelligiblen Formen aufnehmen kann. Dafür bedarf er nun keines Körperorgans. Wenn ein Seelenteil aber keinem Organ entspricht, dann gehört er auch nicht im engeren Sinne zur Entelechie des lebendigen Körpers.

Der Intellekt ist somit unkörperlich und der physischen Welt enthoben. Seine Leistung basiert zwar auf sinnlichen Daten, er ist jedoch um eine Instanz innerlicher als die Sinnesvermögen. Was ihn mit letzteren verbindet, ist seine rezeptive Potentialität. Diese Parallele steht ganz zu Beginn der Abhandlung über den Intellekt: „Wenn nämlich das Denken wie das Wahrnehmen ist, dann ist es wohl entweder eine Art von Erleiden von seiten des Denkgegenstandes oder <vielmehr> etwas anderes von der Art. Leidensunfähig muss es also sein, jedoch aufnahmefähig für die Form und der Möglichkeit nach eines von solcher Art [sc. wie die Form], aber nicht dieses [d.h. die Form in Wirklichkeit], und es muss sich auf gleiche Weise verhalten: wie das Sinnesvermögen zu den Sinnesgegenständen, so die Vernunft zu den Denkgegenständen“. ${ }^{2}$

Gäbe es nun aber nur diesen passiven Intellekt, der alles Intelligible werden kann, dann wäre De anima nicht anschlussfähig an Metaphysik $\wedge$. Zum Glück hat Aristoteles mit dem fünften Kapitel des dritten Buches von De anima die Intellektlehre um das entscheidende Theorem erweitert: um die Lehre vom aktiven Intellekt. Der aktive Intellekt ist reine évépyદıa,

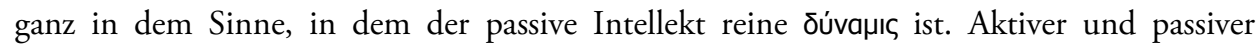
Intellekt sind die beiden Seiten einer substantiellen geistigen Einheit, von der nur die materiale Seite noch irgendwie vom Körper abhängig ist, weshalb sie auch mit ihm vergeht. Sie ist sterblich, und mit ihr sterben alle unsere Kenntnisse und Erfahrungen. Übrig von der ganzen leib-seelischen Einheit, die der Mensch ist, bleibt nur die unvergängliche Seite des aktiven Intellekts. Dieser ist ewig, und unvergänglich und abtrennbar vom Körper. Das meint Aristoteles, wenn er in De anima $\Gamma 5$ sagt: „Und diese Vernunft ist abtrennbar, leidensunfähig und unvermischt, weil sie ihrem Wesen nach Wirklichkeit ist“.3 Auch die

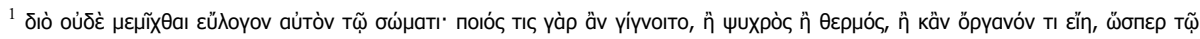
aібӨптוкй (429a24-26).

${ }^{2} \varepsilon$ i ס́

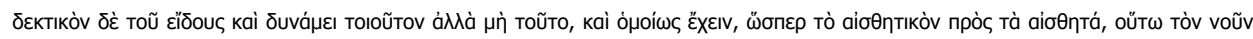
про̀ тà vonтá (429a13-18).

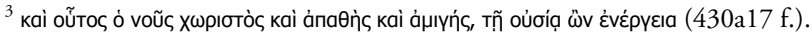


Ewigkeit des aktiven Intellekts formuliert Aristoteles ganz unmissverständlich: „Nur wenn sie aber getrennt ist [sc. vom Körper], ist sie das, was sie ist, und nur dies ist unsterblich und ewig“. ${ }^{4}$ Dieser besondere Charakter des aktiven Intellekts berechtigt Aristoteles denn auch, ihn in De anima als „etwas Göttlicheres“5 zu bezeichnen. In De generatione animalium

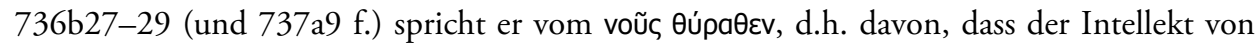
außen in die Seele des Menschen eintritt. Mit der Lehre vom aktiven Intellekt verlässt Aristoteles das Gebiet der Naturphilosophie und vollzieht den Übergang zur Metaphysik. Der aktive Intellekt gehört im Gegensatz zu allen anderen Seelenvermögen nicht mehr in den Naturzusammenhang. Das ist der Sinn des Satzes im Eingangskapitel von De partibus animalium: „Denn auch ist nicht die ganze Seele Natur"6. Ganz besonders deutlich spricht Aristoteles im zehnten Buch der Nikomachischen Ethik davon, dass der menschliche Intellekt göttlich ist. Das Leben der theoretischen Schau übersteigt als Dauerzustand das menschliche Vermögen. Da der Mensch jedoch manchmal an der Schau teilhat, betont Aristoteles, dass dies nicht seiner menschlichen Natur zu verdanken ist, sondern dem Göttlichen in ihm: „Ein solches Leben [sc. der Schau] dürfte aber über das menschliche Vermögen hinausgehen; denn nicht insofern er Mensch ist, wird er so leben, sondern insofern es etwas Göttliches in ihm gibt" ${ }^{\text {"7 }}$. Es ist offenbar, dass er sich hier auf den aktiven Intellekt bezieht, der als einziges Vermögen des Menschen der theoretischen Schau fähig ist. Diese Interpretation wird durch eine weitere Bemerkung aus der Ethik gestützt: „Wenn also der Intellekt etwas Göttliches im Vergleich zum Menschen ist, dann ist auch ein Leben, das sich nach ihm richtet, göttlich im Vergleich zum menschlichen Leben" ${ }^{\text {"8. }}$

Obgleich Aristoteles viele Hinweise darauf gibt, dass der Intellekt, der als göttliches Wesen an der Spitze des Kosmos steht, wesensgleich ist mit dem menschlichen aktiven Intellekt, vollzieht er diese Identifikation doch nicht explizit. Erst Alexander von Aphrodisias tut dies. Ihm folgt ein Strang der arabischen und mittelalterlichen christlichen Tradition. Doch ich denke, dass diese Identifikation der Sache nach erforderlich ist. Nichts weist darauf hin, dass wir etwa eine vermeintlich christliche Vorsicht walten lassen müssen, die Grenzen des Geschöpflichen beim Menschen nicht zu überschreiten und Gott immer eine uneinholbare Überlegenheit zuzugestehen. Nichts gibt uns eine Handhabe, den menschlichen vom göttlichen Intellekt in seinem Wesen zu unterscheiden. Beide müssen wesensmäßig eines sein, wenn anders Intellekt in der Welt obwalten soll.

Es ist ja vielleicht gerade der etwas naive Glaube an die Vernünftigkeit der Welt und das Hingeordnetsein des Menschen auf diese kosmische Ordnung, der uns an der griechischen Philosophie fasziniert. Bei Aristoteles finden wir diesen Glauben jedenfalls noch sehr lebendig. Hierhin gehört auch der Umstand, dass die begriffliche Definition der Dinge mit

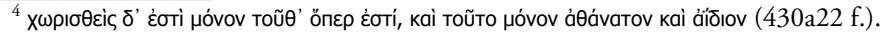

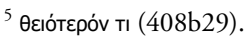

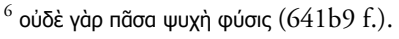

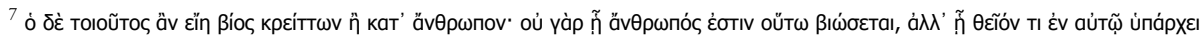
(1177b26-28).

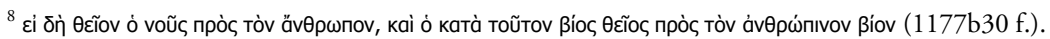


ihrem wesensmäßigen Sein zusammenfällt. Unsere menschliche Erkenntnis und das Wesen der Dinge sind ein- und dasselbe. Erst der Deutsche Idealismus hat die Rangfolge umgekehrt. Bei Aristoteles ist es noch so, dass die Dinge der Welt ihr Sein in unser Bewusstsein einleuchten und dadurch das Intelligible der Welt in unserer Seele Gestalt annimmt. Was liegt also näher, als auch im menschlichen Intellekt, der unser Innerstes ist, eine fundamentale Kongruenz, eine Wesensidentität mit dem höchsten kosmischen Prinzip anzunehmen?

Nachdem ich versucht habe, die Brücke zwischen der menschlichen Seele und Gott im Rahmen des aristotelischen Systems zu schlagen, komme ich zum zweiten Teil meines Referats, in dem ich die Struktur des menschlich-göttlichen Intellekts untersuche.

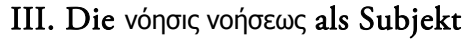

Der Höhepunkt der aristotelischen Metaphysik ist die Gotteslehre des Buches $\Lambda$. Die Metaphysik als die Lehre vom Seienden mündet in das Prinzip des Seienden. Die Metaphysica generalis kulminiert in der Metaphysica specialis, der Theologie, wie Aristoteles sie selbst

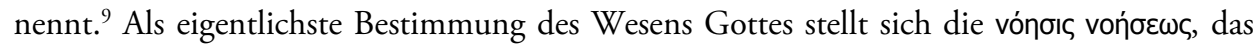
Denken des Denkens heraus. Wie Aristoteles zu diesem Ausdruck kommt und was er damit meint, will ich nun versuchen, in möglichster Prägnanz darzulegen.

Es gibt im Buch $\Lambda$ der Metaphysik, vor allem in den Kapiteln 7 und 9, zwei Hauptstränge der Argumentation. Der erste sucht nach einer Letztbegründung der Bewegung, der zweite stellt Gott, den Ersten Beweger, als höchsten Intellekt dar. Das erste Argument nenne ich

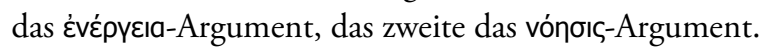

Das źvépyદıa-Argument steht vollkommen im Zusammenhang der aristotelischen Physik, deren Hauptthema die Bewegung ist. Im Gegensatz zu Platon ist Aristoteles sehr an einer Letztbegründung der Bewegung interessiert. Und auch wenn sie ihm letzten Endes nicht gelingt, so ist doch das Auffinden eines ersten Prinzips von Bewegung das Motiv, das ihn dazu führt, einen ersten Beweger anzunehmen, der als Gott im Sinne einer natürlichen Theologie angesprochen werden kann. ${ }^{10}$ Entscheidend für das Argument ist die Notwendigkeit, einen Begründungsregress zu vermeiden. Denn alles, was bewegt ist, wird von anderem bewegt und kann nur eine mittlere Stellung in der Kette der Bewegungsvermittlung einnehmen. Es muss etwas geben, das zwar anderes bewegt, aber nicht wieder bewegt wird. Dies ist das primum movens ipsum non motum, der Erste Beweger. Dieser Erste Beweger bewegt nun die ewige Kreisbewegung der Fixsternsphäre, deren Bewegung so nah an die absolute Vollkommenheit Gottes herankommt, wie es für materiell Seiende nur möglich ist. Und doch braucht sie aus der Perspektive der aristotelischen Substanzontologie ein Substrat, das sich nicht bewegt und ebenfalls ewig ist. Eine solches Substrat kann nur eine ewige Substanz sein, die ihrerseits aus reiner Aktivität bestehen muss. Denn jede Passivität bzw. Potentialität würde den Bestand des Kosmos gefährden. Also ist der Erste Beweger, der die Fixsternsphäre in

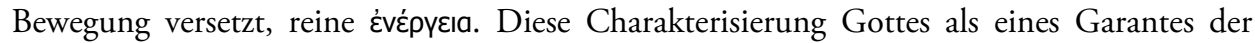

\footnotetext{
${ }^{9}$ In Met. E 1, 1026a29 und K 8, 1064 b3.

${ }^{10} \mathrm{Vgl}$. Met. $1072 \mathrm{~b} 25$.
} 
Beständigkeit der in Bewegung befindlichen Welt ist noch ganz physikalisch und äußerlich gedacht. Das vónoıs-Argument dringt nun weiter vor, indem es die innere Verfasstheit dieses Gottes untersucht.

Gott als der Erste Beweger bewegt ganz anders als alle anderen Bewegenden. Denn für die Übermittlung von Bewegung ist ein gewisser physischer Kontakt erforderlich. Dies kann für Gott nicht gelten. Er muss auf eine seiner Natur angemessene Weise bewegen. Aristoteles sagt, er bewege als Begehrtes und Gedachtes. Der ganz Kosmos strebt danach, dieses Streben Vollkommenheit der źvépyદıa Gottes nachzuahmen. Er strebt also zu Gott hin, und dies versetzt alles in Bewegung. Das Begehrte ist aber ein als Begehrtes Erkanntes, d.h. die vónбıৎ des Begehrten geht dem Begehren selbst als dessen Bedingung voraus. Somit ist Gott

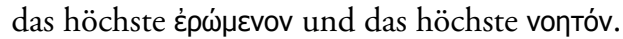

Wir Menschen haben mehr Lust am Tätigsein als am Untätigsein. Das gilt auch für Gott, dessen Lebensform die höchste im Kosmos ist. Er ist reine lustvolle Lebendigkeit und daher auch reine Tätigkeit. Was ist aber die höchste Form der Tätigkeit? Schon für uns Menschen ist dies das Denken, wie Aristoteles im zehnten Buch der Nikomachischen Ethik betont. Gott kann nun gar nichts anderes sein als Denken. Denn er hat keinen Körper und ist nicht in der Zeit. All dies würde Materialität oder doch zumindest Potentialität erfordern, die in ihm als actus purus nicht sein können. Seine Tätigkeit muss also reine vónбıৎ sein; er selbst ist reiner voũc.

Es ist nun nur noch ein kleiner Schritt zur Etablierung des Wesens Gottes als vónoıৎ

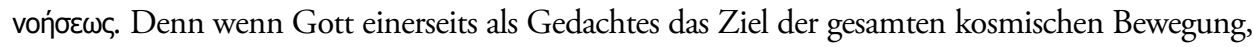
andererseits seinem lebendigen Wesen nach selbst Denken ist, dann kann die Frage, was Gott eigentlich denkt, nur noch so beantwortet werden: Er denkt sich selbst, weil alle anderen Gegenstände des Denkens ihm selbst untergeordnet sind. Dächte Gott etwas anderes als sich selbst, dann wäre dieses Gedachte höherrangig (тіні́́тероv) als er selbst und er mithin nicht das höchste Denkbare. Also bleibt nur eine Lösung: Gott als lebendiger Intellekt ist das Denken

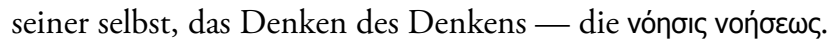

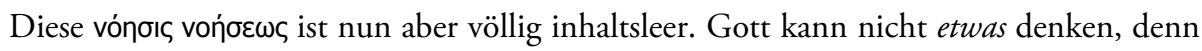
jedes Gedachte impliziert eine gewisse Potentialität. So ist es nämlich bei uns Menschen: Der passive Intellekt ist das Gefäß für die intelligiblen Formen der Dinge der Welt. Insofern wir etwas denken, verhalten wir uns dem Gedachten gegenüber rezeptiv. Das ist bei Gott

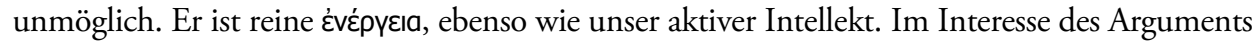
für eine Theorie der Subjektivität habe ich, über den Wortlaut des Aristoteles hinausgehend, den menschlichen aktiven Intellekt mit dem offenbar wesensgleichen göttlichen Intellekt

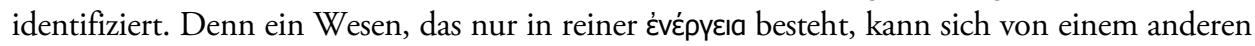

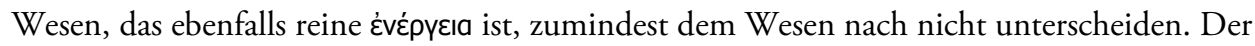
Frage nach der Möglichkeit numerischer Verschiedenheit kann ich hier nicht nachgehen. Sie ist aber auch nicht erforderlich, da es hier nur um die Struktur der Subjektivität und nicht um die Anzahl der Subjekte geht.

Dieses Denken des Denkens kann nun nichts mit der von Aristoteles behaupteten Reflexivität der Sinnlichkeit und des Denkens als Begleiterscheinung zu tun haben, die sich 


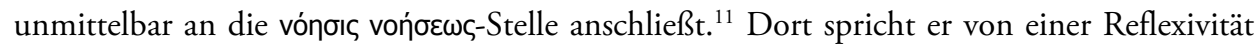

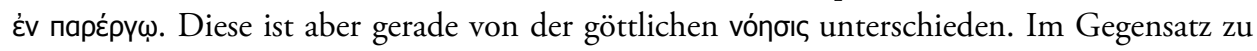
diesem begleitenden Selbstwissen bzw. -wahrnehmen ist das Selbstwissen Gottes ein direktes und ausschließliches. Es kann nicht an der Erkenntnis anderer Gegenstände stattfinden, sondern nur in seiner ganzen Reinheit.

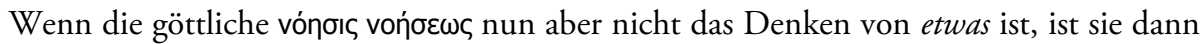
überhaupt eine Reflexivstruktur? Diese Frage führt uns nun zum zentralen Anliegen meines Referats. Die neuzeitlichen Versuche, Subjektivität als Selbstbewusstsein zu fassen, sind an der Objektivierungsstruktur des intentionalen Bewusstseins gescheitert. Denn ein Subjekt das sich selbst zu erfassen versucht, kann sich nur als Objekt seiner Denktätigkeit erfassen. Als Objekt ist es aber nicht mehr das Subjekt, als das es sich erfassen wollte. Am wenigsten gerät Kant in diese Regressgefahr. Seine transzendentale Subjektivität ist klar vom empirischen Selbstbewusstsein unterschieden. Es ist die reine Bedingung subjektzugehöriger Denkgehalte.

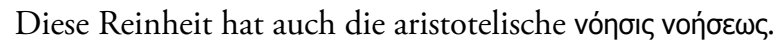

Ich gehe davon aus, dass die sprachliche Form dieses Ausdrucks ein Hinweis auf die Unmöglichkeit ist, Subjektivität von einem intentionalen Bewusstsein abzuleiten. Das Denken des Denkens ist also kein selbstobjektivierender Bewusstseinsakt, der sich selbst als Objekt in den Zusammenhang der Erfahrung einreiht, sondern die Abwesenheit von Intentionalität, ein Denken von nichts in einem so prägnanten Sinne, dass das Denken selbst in seiner Struktur hinterfragt wird. Gott ist zwar reine Aktualität und als diese reine Aktualität ein geistiger Vollzug. Aber die Abwesenheit jeder Potentialität in ihm deutet darauf hin, dass auch das fehlen muss, was wir als Gerichtetheit des Denkens anzunehmen gewohnt sind. Es bleibt ein Denken in einer Reinheit, die sich unserer Vorstellungskraft

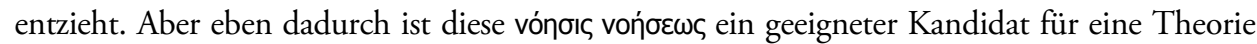
der Subjektivität, die sich jedes Ballastes entledigen möchte, der ihr vom objektivierenden Bewusstsein aufoktroyiert wird. Diese Subjektivität wäre ein wahrhaft reines Ich. Denn der Gedanke „Ich“ ist ja in der Tat völlig gehaltlos. Er offenbart die geistige Instanz, die die Grundbedingung dafür ist, dass wir uns Erfahrungen und Gedanken zuschreiben können. Der Gedanke „Ich“ ist bei sich, ohne zu sich zurückkehren zu müssen. Denn er ist niemals ausgegangen.

Ich merke, dass sich meine sprachlichen Formulierungen den Predigten Meister Eckharts annähern. Vielleicht ist dies das Zeichen, dass ich zum Ende kommen sollte jedenfalls was die Subjektivität bei Aristoteles betrifft. Fortgesetzt wurde diese Theorie aber in der Tat in den Intellektspekulationen des Mittelalters, und von da aus sind die Traditionslinien zur Neuzeit recht gut bekannt. Was ich hoffe gezeigt zu haben, ist, dass Aristoteles zumindest ein legitimer Ausgangspunkt der Entwicklung der Selbstbewusstseinstheorien ist und dass sein Theorievorrat ein anderes Modell von Subjektivität als das der Neuzeit ermöglicht.

\footnotetext{
${ }^{11}$ Met. $\wedge 9,1074 \mathrm{~b} 35 \mathrm{f}$.
} 